\title{
Binding of Straight-Chain Saturated Dicarboxylic Acids to Albumin
}

\author{
James H. Tonsgard, ${ }^{*}$ Stephen A. Mendelson, ${ }^{*}$ and Stephen C. Meredith ${ }^{5}$ \\ Departments of ${ }^{* \ddagger}$ Pediatrics, ${ }^{*}$ Neurology, and ${ }^{\S}$ Pathology, and ${ }^{*}$ the Joseph Kennedy Mental Retardation Center, Pritzker Medical \\ School, The University of Chicago, Chicago, Illinois 60637
}

\begin{abstract}
Dicarboxylic acids are prominent features of several diseases, including Reye's syndrome. Long-chain dicarboxylic acids have profound effects on the function and structure of isolated mitochondria, suggesting that they could contribute to the mitochondrial dysfunction in Reye's syndrome. Binding of fatty acids to albumin and the intracellular fatty acid-binding proteins is important in regulating the transport and metabolism of fatty acids and protects against the toxic effects of unbound fatty acids. We studied the binding of dicarboxylic acids to defatted albumin using equilibrium dialysis to assess to what extent dicarboxylic acids are likely to be bound in the plasma of patients. Dicarboxylic acids bind weakly to albumin in a molar ratio of $3.8,4.2,1.6,0.8$, and 0.7 to 1 for octadecanedioic, hexadecanedioic, tetradecanedioic, dodecanedioic, and decanedioic acid, respectively. The dissociation constants for long-chain dicarboxylic acids are 100-1,000-fold larger than those of comparable monocarboxylic acids. Oleate competes with dicarboxylic acid and reduces the moles of dicarboxylic acid bound per mol of albumin to $<1$. Octanoate inhibits dicarboxylic acid binding. Our observations indicate that in Reye's syndrome, substantial concentrations of dicarboxylic acids of patients may be free and potentially toxic to mitochondria and other cellular processes.
\end{abstract}

\section{Introduction}

Dicarboxylic acids are the product of omega-oxidation in the microsomes. Only 5-10\% of FFA are metabolized by this pathway in ketotic rats (1). However, dicarboxylic acids have recently been shown to be a prominent feature of several diseases, including Reye's syndrome (2-4), neonatal adrenoleukodystrophy (5), Zellweger's syndrome (5), and defects of fatty acid metabolism $(6,7)$. In Reye's syndrome, dicarboxylic acids make up as much as $55 \%$ of the total serum FFA (4).

The hallmark of Reye's syndrome is a transient generalized impairment of mitochondrial enzymes and swelling and distortion of mitochondrial ultrastructure (8). Several toxins, including aspirin, have been suggested to predispose children to this illness (9). Serum from patients with Reye's syndrome impairs ATP formation and induces swelling and distortion of isolated mitochondria (10). These effects correlate directly

Address all correspondence to Dr. James $\mathrm{H}$. Tonsgard, Department of Pediatrics, Box 228, The University of Chicago, 5841 S. Maryland Avenue, Chicago, IL 60637.

Received for publication 20 January 1988 and in revised form 13 June 1988.

J. Clin. Invest.

(C) The American Society for Clinical Investigation, Inc.

$0021-9738 / 88 / 11 / 1567 / 07 \quad \$ 2.00$

Volume 82, November 1988, 1567-1573 with the concentration of dicarboxylic acids in the serum samples (10). Studies with isolated mitochondria have demonstrated that dicarboxylic acids, particularly long-chain dicarboxylic acids, at concentrations comparable to those in plasma of patients with Reye's syndrome, profoundly inhibit the enzymes of the terminal respiratory pathway (11), inhibit ATP formation (10), and induce an irreversible expansion of mitochondria characteristic of an uncoupler of oxidative phosphorylation (12). Dicarboxylic acids thus may contribute to the mitochondrial dysfunction that is central to Reye's syndrome (10). The regulation of dicarboxylic acid metabolism may therefore be important in Reye's syndrome and other diseases in which dicarboxylic acid formation is prominent.

The binding of monocarboxylic fatty acids to albumin and the intracellular fatty acid-binding proteins modulates the transport and metabolism of these fatty acids $(13,14)$. Binding of potentially toxic ligands to albumin protects against the toxic effects of the ligands, as toxicity is proportionate to the concentration of unbound ligand $(13,15,16)$. We undertook this study to determine the affinity and capacity of albumin for dicarboxylic acids.

\section{Methods}

Monocarboxylic and dicarboxylic acids. Unlabeled monocarboxylic and dicarboxylic acids were purchased from Sigma Chemical Co. (St. Louis, MO), Applied Science (Warrenville, IL), and Foxboro Analabs (North Haven, CT). The purity of the unlabeled acids was assessed by gas-liquid chromatography and was found to be at least $99.5 \%$. $\left[{ }^{3} \mathrm{H}\right]-$ Dicarboxylic acids, $1-\left[{ }^{14} \mathrm{C}\right] \mathrm{C}_{8.0},{ }^{1}$ and $1-\left[{ }^{14} \mathrm{C}\right] \mathrm{C}_{18.1}$ were purchased from Amersham Corp. (Arlington Heights, IL) and 1- $\left[{ }^{14} \mathrm{C}\right]$ decanedioic acid was purchased from Pathfinder Laboratories, Inc. (St. Louis, MO). The $\left[{ }^{3} \mathrm{H}\right]$ dicarboxylic acids prepared by Amersham Corp. were obtained by reaction with tritiated water (method TR.8; Amersham International, Amersham, England) at a very high specific activity. The chemical purity of the labeled compounds was assessed by gas-liquid chromatography (3) and found to be at least $97 \%$. The radiopurity of the labeled compounds was assessed by thin-layer chromatography (17). Silica $G$ plates were spotted with a mixture of labeled and unlabeled methyl esters of the dicarboxylic acids, separated using pentane/ ether/acetic acid (92:7:1), and visualized with iodine vapors. The plates were scraped in 1-cm bands and the gel fractions were solubilized with water and then the radioactivity was measured. The radiopurity of the dicarboxylic acids was at least $\mathbf{9 3 . 4 \%}$.

Potassium salts of a mixture of labeled and unlabeled monocarboxylic or dicarboxylic acid were made by dissolving the acid in a small amount of ethanol and adding $10 \mathrm{meq}$ of $1 \mathrm{M}$ methanolic $\mathrm{KOH}$ to 1 meq acid. Phenolphthalein was added as a pH indicator. The flask containing the solution was connected to a refluxing apparatus and heated at $80^{\circ} \mathrm{C}$ for $30 \mathrm{~min}$ in a water bath. The solution was cooled, then dried with $\mathrm{N}_{2}$, and then redissolved in phosphate buffered solution containing $0.116 \mathrm{M} \mathrm{NaCl}, 0.0049 \mathrm{M} \mathrm{KCl}$, and $0.016 \mathrm{M}$ sodium

1. Abbreviations used in this paper: $\mathrm{C}_{18.1}$, oleic acid; $\mathrm{C}_{18.0}$, stearic acid; $C_{16.0}$, palmitic acid; $C_{14.0}$, myristic acid; $C_{12.0}$, lauric acid; $C_{10.0}$, decanoic acid; $\mathrm{C}_{8.0}$, octanoic acid. 
phosphate, $\mathrm{pH}$ 7.4. This salt solution was rinsed with chloroform to remove the monocarboxylic or dicarboxylic acids that were not in salt form. The concentration of the carboxylate salt was determined by gas-liquid chromatography by comparing the detector response to a calibration curve of a known amount of the unlabeled compound. The extraction efficiency was determined by comparing the radioactivity of aliquots before and after extraction. The specific activity of the carboxylate salt was determined from the counts per minute of the extracted, derivatized sample divided by the concentration of the carboxylate salt.

Albumin. Essentially fatty acid-free $(<0.005 \%)$ crystalline BSA was from Sigma Chemical Co. and further purified as described by Spector, John, and Fletcher (18). The monocarboxylic fatty acid content of the purified albumin was determined by gas-liquid chromatography (4) and found to have $<0.02 \mathrm{~mol}$ fatty acid/mol of albumin.

Equilibrium dialysis. Binding of dicarboxylic acids to defatted BSA was determined using equilibrium dialysis as described by Ashbrook, Spector, and Fletcher (19). Dialysis chambers and membranes were from Bel-Art Products (Pequannok, NJ). The chamber contains two $1-\mathrm{ml}$ compartments separated by a dialysis membrane that is impermeable to compounds with a molecular weight $>6,000$; albumin does not cross the dialysis membrane (19). The dicarboxylic acids reached equilibrium within $18 \mathrm{~h}$ at $37^{\circ} \mathrm{C}$. We chose a 26-h incubation period to ensure equilibrium. Binding was assessed by varying concentrations of dicarboxylic acid $(0.05-1.5 \mathrm{mM})$ in a near physiologic salt solution containing $0.116 \mathrm{M} \mathrm{NaCl}, 0.0049 \mathrm{M} \mathrm{KCl}$, and $0.016 \mathrm{M}$ sodium phosphate, $\mathrm{pH}$ 7.4. Dicarboxylic acid was added to one side of the chamber and defatted albumin in the same salt solution was added to the other side to a final concentration of $0.05 \mathrm{mM}$. Because octadecanedioic acid is less soluble than the other dicarboxylic acids (20), binding of this fatty acid was assessed using $0.010 \mathrm{mM}$ albumin and $0.010-0.200 \mathrm{mM}$ octadecanedioic acid. Dialysis chambers were incubated in a shaking water bath at $37^{\circ} \mathrm{C}$ and at the end of the incubation period, $100 \mu \mathrm{l}$ was removed from each side, and the radioactivity of the aliquot was measured using an Isocap/300 liquid scintillation counter (G. D. Searle \& Co., Des Plaines, IL). The concentration of free dicarboxylic acid was determined from the counts per minute in the aliquot from the side of the chamber that did not contain albumin, corrected for the volume of the two sides of the chamber into which the free dicarboxylic acid was distributed, and multiplied by the specific radioactivity of the dicarboxylic acid solution. The concentration of bound dicarboxylic acid was determined by subtracting the counts per minute in the aliquot from the side of the equilibrium chamber that did not contain albumin, from the counts per minute in the aliquot from the albumincontaining side. This was then corrected for the volume of the albumin-containing chamber and multiplied by the specific radioactivity of the dicarboxylic acid solution. Experiments were performed in triplicate. The recovery of radioactivity was between 88 and $101 \%$. The data shown are the results of between four and eight separate experiments.

Monocarboxylic acid competition. Experiments involving the competition of the $1-\left[{ }^{14} \mathrm{C}\right] \mathrm{C}_{18.1}$ with ${ }^{3} \mathrm{H}$-labeled dicarboxylic acids were performed as described by Ashbrook, Spector, and Fletcher (19). It has previously been observed that $C_{18.1}$ does not readily cross the dialysis membrane $(15,19)$. This observation was confirmed in preliminary experiments. The competition experiments were performed as described above, except that either $0.1,0.3$, or $0.5 \mathrm{mM} 1-\left[{ }^{14} \mathrm{C}\right] \mathrm{C}_{18.1}$ was added to the albumin-containing side of the equilibrium chamber. The extent to which $\mathrm{C}_{18.1}$ was bound was determined by gel filtration (21, 22). At the end of the incubation, $100 \mu \mathrm{l}$ was removed from the albumin-containing side of the chamber and added to a 1-ml Sephadex G-25 column (Pharmacia Fine Chemicals, Piscataway, NJ). The column was prepared by placing a slurry of Sephadex G-25 in buffered salt solution in a $1-\mathrm{ml}$ plastic syringe. The buffer was removed by centrifugation at $150 \mathrm{~g}$ for $10 \mathrm{~min}$ at $25^{\circ} \mathrm{C} .100 \mu \mathrm{l}$ from the albumin side of the chamber was added to the column and the column was centrifuged again at $150 \mathrm{~g}$ for $10 \mathrm{~min}$. Protein content of the eluate was monitored spectrophotometrically at $280 \mathrm{~nm}$ and fatty acid content was assessed by scintillation counting. At least $90 \%$ of the albumin is eluted after a single centrifugation. Albumin was completely eluted from the column with two additional rinses of $100 \mu$ l of salt solution. Fatty acids not associated with albumin remained bound to the column when the column was eluted with additional rinses of aqueous buffer. Recovery of the FFA was achieved by rinsing the column three times with $0.5 \mathrm{ml}$ of $2: 1$ chloroform/methanol. The buffer and chloroform rinses were collected separately and transferred to scintillation vials. The samples were evaporated to dryness and the amount of $\left[{ }^{14} \mathrm{C}\right] \mathrm{C}_{18.1}$ was determined by liquid scintillation. The mean recovery of albumin was determined to be $95.8 \%$. The mean recovery of $\left[{ }^{14} \mathrm{C}\right] \mathrm{C}_{18.1}$ was $98.3 \%$. We demonstrated that reequilibration of bound fatty acid is sufficiently slow that it does not occur to any significant extent, as albumin is propelled by centrifugation through the column.

In contrast to $C_{18.1}, C_{8.0}$ freely diffuses across the dialysis membrane (19). Equilibration of $\mathrm{C}_{8.0}$ occurs within $24 \mathrm{~h}$. Therefore, binding of $\left[{ }^{14} \mathrm{C}\right] \mathrm{C}_{8.0}$ was assessed from the partitioning of ${ }^{14} \mathrm{C}$ counts per minute as described previously for the dicarboxylic acids.

Analysis of binding. Binding isotherms were analyzed using a nonlinear least-squares fit computer program; in general, the damping Gauss-Newton method was used (23). The points in the figures are experimental data and the lines are the best fit of the data to the theoretical equation as determined by the least-squares fit computer program. Dissociation constants are expressed as the mean $\pm \mathrm{SD}$.

\section{Results}

The binding of dicarboxylic fatty acids to albumin was analyzed using the equation of reversible, saturable binding to one, two, or three classes of noninteracting equivalent sites, i.e., the equation of a Langmuir isotherm (24). For the case of one class of sites, $K_{\mathrm{d}}=\left(F A_{\mathrm{f}}\right)\left(S_{\mathrm{f}}\right) / C_{\mathrm{x}}=\left(F A_{\mathrm{f}}\right)\left(S_{\mathrm{t}}-F A_{\mathrm{b}}\right) / F A_{\mathrm{b}}$, where $K_{\mathrm{d}}=$ dissociation constant (micromolar), $F A_{\mathrm{f}}=$ free dicarboxylic acid (micromolar), $F A_{\mathrm{b}}=$ bound dicarboxylic acid (micromolar), $\mathrm{C}_{\mathrm{x}}=$ dicarboxylic acid-site complex (micromolar), and $S_{\mathrm{f}}$ and $S_{\mathrm{t}}=$ free and total binding sites, respectively. The number of sites/albumin molecule can be calculated from $S_{\mathrm{t}}$ and the albumin concentration. We used the Akaike information coefficient (23) to ascertain whether the best fit of the data could be obtained by assuming one, two, or three classes of binding sites.

Affinity of dicarboxylic acids for albumin. We first examined the binding of hexadecanedioic acid (Fig. 1). Analysis of the binding shows two different types of binding sites with dissociation constants \pm SD of $1.2 \pm 1.4$ and $67.8 \pm 15.6 \mu \mathrm{M}$, respectively (Table I). The first site binds $1 \mathrm{~mol}$ of dicarboxylic acid and the second class of binding sites binds the remaining acid. We observed a molar ratio of bound hexadecanedioic acid to albumin of 4.2:1. The calculated saturation of albumin occurs at a molar ratio of $5: 1(0.9 \pm 0.3$ plus $4.2 \pm 0.2 \mathrm{~mol}$, Table I).

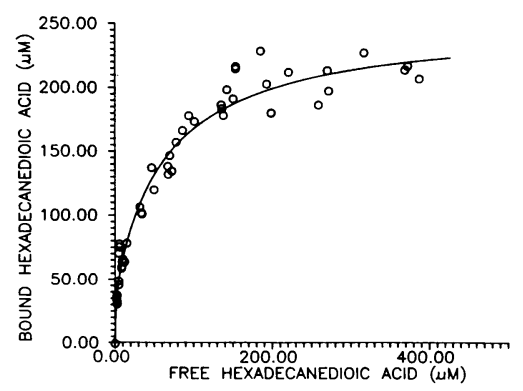

Figure 1. Binding of hexadecanedioic acid to defatted albumin. Binding was assessed at $\mathrm{pH}$ 7.4, using varying concentrations of $\left[{ }^{3} \mathrm{H}\right]-$ dicarboxylic acid in an equilibrium dialysis chamber in which one side contained 0.050 $\mathrm{mM}$ defatted albumin. The points are experimental data and the lines are the best fit of the data to the theoretical equation. 


\begin{tabular}{|c|c|c|c|c|c|c|}
\hline \multirow[b]{2}{*}{ Fatty acid } & \multirow{2}{*}{$\begin{array}{l}\text { Bound } \\
\text { dicarboxylic } \\
\text { acid }\end{array}$} & \multicolumn{2}{|c|}{ Dissociation constants } & \multicolumn{2}{|c|}{ Predicted saturation } & \multirow[b]{2}{*}{ Number of data points } \\
\hline & & $K_{d_{1}}$ & $K_{d z}$ & Site 1 & Site 2 & \\
\hline & $\mathrm{mol} / \mathrm{mol} \mathrm{BSA} A^{*}$ & \multicolumn{2}{|c|}{$\mu M \pm S D$} & \multicolumn{2}{|c|}{$\mathrm{mol} / \mathrm{mol} B S A \pm S D$} & \\
\hline \multicolumn{7}{|c|}{ Octadecanedioic } \\
\hline pH 7.4 & 3.8 & $1.1 \pm 1.7$ & $19.4 \pm 18.2$ & $1.4 \pm 1.4$ & $3.2 \pm 1.1$ & 28 \\
\hline \multicolumn{7}{|c|}{ Hexadecanedioic } \\
\hline pH 6.8 & 4.2 & $2.5 \pm 1.4$ & $27.8 \pm 6.1$ & $1.1 \pm 0.4$ & $3.3 \pm 0.3$ & 35 \\
\hline pH 7.4 & 4.2 & $1.2 \pm 1.4$ & $67.8 \pm 15.6$ & $0.9 \pm 0.3$ & $4.2 \pm 0.2$ & 51 \\
\hline pH 8.0 & 4.2 & $3.9 \pm 0.9$ & $216.0 \pm 46.0$ & $2.1 \pm 0.2$ & $4.3 \pm 0.9$ & 41 \\
\hline \multicolumn{7}{|c|}{ Tetradecanedioic } \\
\hline pH 7.4 & 1.6 & $23.2 \pm 9.8$ & $292.6 \pm 26.0$ & $1.0 \pm 0.1$ & $1.1 \pm 0.1$ & 49 \\
\hline \multicolumn{7}{|l|}{ Dodecanedioic } \\
\hline pH 6.8 & 0.80 & & $30.1 \pm 6.2$ & & $0.9 \pm 0.1$ & 22 \\
\hline pH 7.4 & 0.88 & & $75.2 \pm 4.3$ & & $1.0 \pm 0.02$ & 28 \\
\hline $\mathrm{pH} 8.0$ & 0.80 & & $83.3 \pm 13.2$ & & $1.0 \pm 0.1$ & 21 \\
\hline \multicolumn{7}{|l|}{ Decanedioic } \\
\hline pH 7.4 & .70 & & $31.5 \pm 9.3$ & & $0.8 \pm 0.1$ & 17 \\
\hline
\end{tabular}

Equilibrium constants for saturated straight-chain dicarboxylic acids. Binding was assessed in the presence of $0.050 \mathrm{mM}$ albumin for all of the dicarboxylic acids except octadecanedioic acid, which was incubated with $0.010 \mathrm{mM}$ albumin. ${ }^{*}$ Indicates the mol ratio of fatty acid to albumin at saturation as observed experimentally.

The binding of octadecanedioic acid is similar to that of hexadecanedioic acid (Fig. 2). Octadecanedioic acid is bound in a 3.8:1 molar ratio (Table I). As with hexadecanedioic acid, there appear to be two distinct types of binding sites with a theoretical saturation of $4.6 \pm 1.4 \mathrm{~mol}$ of the dicarboxylic acid.

In contrast to the longer chain dicarboxylic acids, albumin only binds $2 \mathrm{~mol}$ of tetradecanedioic acid per mol of albumin (Table I, Fig. 2). There are again two distinct binding sites, each binding $1 \mathrm{~mol}$ of tetradecanedioic acid per mol of albumin with dissociation constants of $23.2 \pm 9.8$ and $292.6 \pm 26.0$ $\mu \mathrm{M}$ (Table I). There is only a single binding site for dodecanedioic and decanedioic acids. The maximal binding achieved

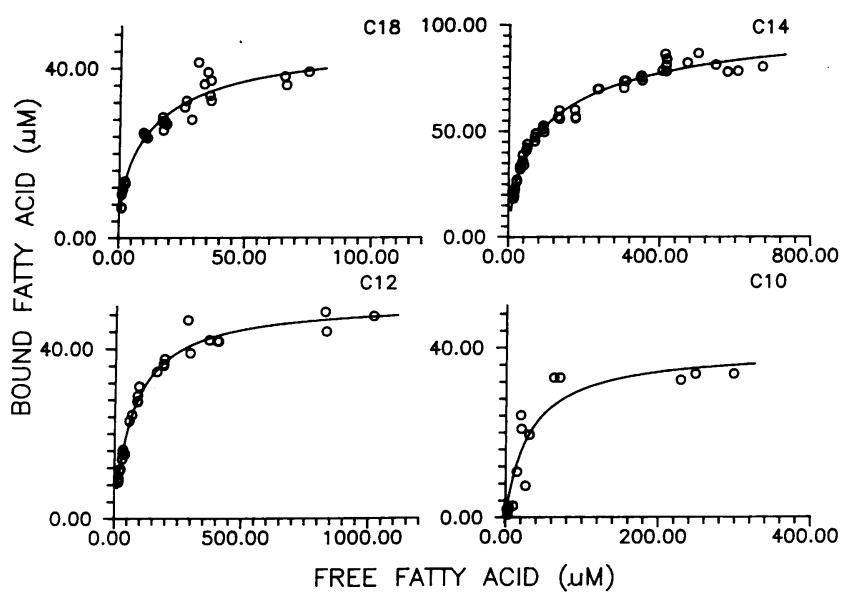

Figure 2. Binding of dicarboxylic acids to defatted albumin. Binding was assessed as described in Fig. 1 with the exception that the binding of octadecanedioic acid was assessed with $0.010 \mathrm{mM}$ albumin. Octadecanedioic acid (C18), tetradecanedioic acid (C14), dodecanedioic acid (C12), and decanedioic acid (C10). experimentally for dodecanedioic and decanedioic acids is $\mathbf{0 . 8}$ and $0.7 \mathrm{~mol} / \mathrm{mol}$ of albumin (Fig. 2, Table I). The dissociation constants for dodecanedioic and decanedioic acid are $75.2 \pm 4.3$ and $31.5 \pm 9.3 \mu \mathrm{M}$, respectively.

Effect of $\mathrm{pH}$ on dicarboxylic acid binding. The effect of $\mathrm{pH}$ of the buffer solution on dicarboxylic acid binding was also examined. Incubations were performed at $\mathrm{pH} 6.8$ and 8.0 with hexadecanedioic acid and dodecanedioic acid and compared with incubations at pH 7.4 (Table I). The pH of the buffer solution does not affect the saturation level of the albumin. The binding curves for both hexadecanedioic and dodecanedioic acid shift slightly to the left with a more acidic pH (Fig. 3 ). The $\mathrm{pH}$ of the buffer has no significant effect on the $K_{\mathrm{d} 1}$ for hexadecanedioic acid but the $K_{\mathrm{d} 2}$ decreases at the more acidic $\mathrm{pH}$. The dissociation constant for dodecanedioic acid also decreases at pH 6.8 (Table I).

Competition with monocarboxylic fatty acids for binding. We examined the effect of the monocarboxylic acid, oleic acid $\left(C_{18.1}\right)$ on the binding of dicarboxylic acids using $0.1,0.3$, and $0.5 \mathrm{mM} \mathrm{C}_{18.1}$ (Fig. 4, Table II). $\mathrm{C}_{18.1}$ competes with hexadecanedioic acid for binding; as a result, the apparent dissociation constant of the dicarboxylic acid increases 15-35-fold (from $1.2 \pm 1.4$ to $41.0 \pm 7.3 \mu \mathrm{M}$, Table II). When the monocarboxylic acid concentration is increased to $0.5 \mathrm{mM}$ (a 10:1 $\mathrm{C}_{18.1}$ /albumin ratio), all but $0.70 \mathrm{~mol}$ of hexadecanedioic acid is displaced (Fig. 4, Table II). A similar result was obtained when the binding of octadecanedioic acid was examined in the presence of $\mathrm{C}_{18.1}$. All but $0.7 \mathrm{~mol}$ of octadecanedioic acid is displaced in the presence of near saturating concentrations of $C_{18.1}$. As shown in Fig. 5, when the concentration of long chain dicarboxylic acid is high enough, long-chain dicarboxylic acid can bind to albumin and displace some $C_{18.1}$.

The effect of monocarboxylic fatty acids on the binding of dodecanedioic acid was also examined (Fig. 6, Tables II and 
A

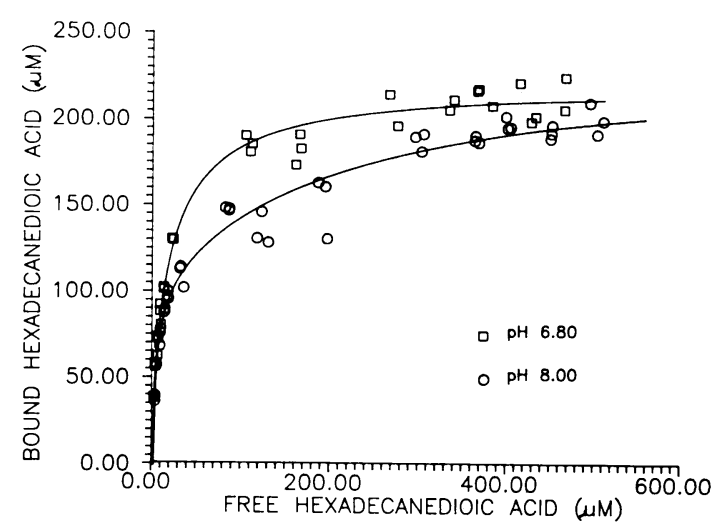

B

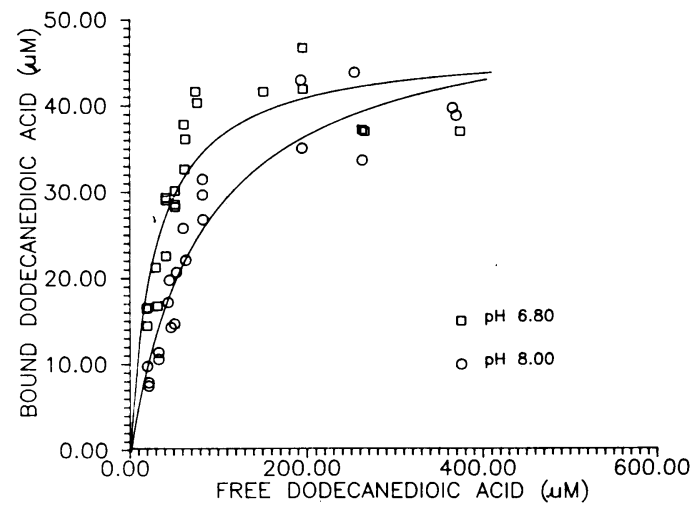

Figure 3. The effect of buffer $\mathrm{pH}$ on the binding of dicarboxylic acids. Binding of hexadecanedioic acid $(A)$ and dodecanedioic acid $(B)$ was assessed at pH $6.8(\square)$ and $8.0(0)$ as described in Fig. 1.

III). In the absence of any competition, between 0.8 and 0.9 mol are bound per mol of albumin. When 2-3 mol of $\mathrm{C}_{18.1}$ are bound to albumin $\left(0.10-0.15 \mathrm{mM} \mathrm{C}_{18.1}\right)$, the affinity for dodecanedioic acid is reduced almost fivefold (Table II), but there is no significant effect on the maximal binding capacity (Tables II and III). When 4-5 mol of $C_{18.1}$ are bound to albumin $\left(0.3-0.5 \mathrm{mM} \mathrm{C}_{18.1}\right)$ the affinity is reduced ninefold (Table II)

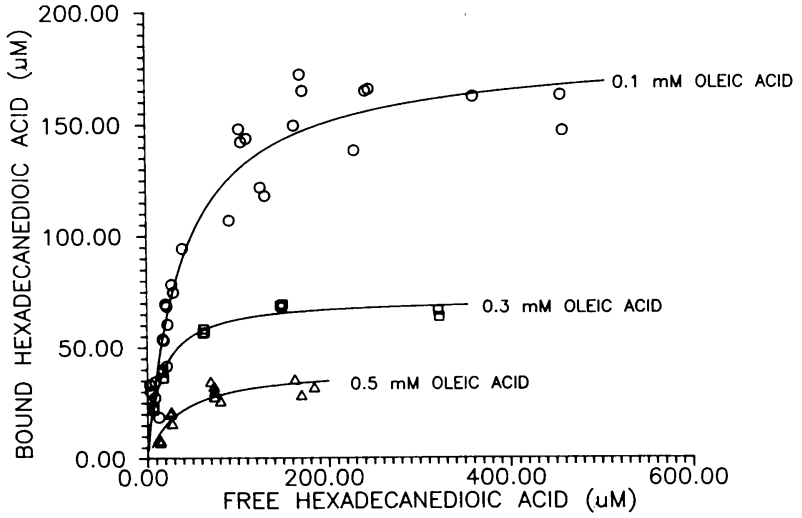

Figure 4. Competition of oleic acid and hexadecanedioic acid for binding to albumin. Binding of $\left[{ }^{3} \mathrm{H}\right]$ dicarboxylic acid was assessed in the presence of $0.05 \mathrm{mM}$ albumin incubated with $0.1(0), 0.3(\square)$, and $0.5(\Delta) \mathrm{mM}\left[{ }^{14} \mathrm{C}\right]$ oleic acid.

and the experimentally observed binding capacity of dodecanedioic acid is reduced by more than half (Table III).

The medium-chain length monocarboxylic acid, octanoic acid $\left(\mathrm{C}_{8.0}\right)$ has a more profound effect on the binding of dodecanedioic acid (Table III). When $0.5 \mathrm{~mol}$ of $\mathrm{C}_{8.0}$ per mol of albumin are bound, only $0.1-0.2 \mathrm{~mol}$ of dodecanedioic acid is bound and when $1 \mathrm{~mol}$ of $\mathrm{C}_{8.0}$ per mol of albumin is bound, the binding of dodecanedioic acid is further inhibited.

\section{Discussion}

Our results indicate that dicarboxylic acids bind to albumin with lower affinity than monocarboxylic acids of the same chain length. There is a single low-affinity site for dodecanedioic and decanedioic acid. There is a single higher affinity site for the longer chain dicarboxylic acids (C18-C14) with between one and four additional low-affinity sites, depending on the chain length of the dicarboxylic acid. The affinity of longchain dicarboxylic acids for albumin is 100-1,000-fold less than that of long-chain monocarboxylic fatty acids and is more comparable to the affinity of many drugs for albumin (25). The molar ratio of bound dicarboxylic acid to albumin observed is also significantly less than that reported for monocar-

Table II. Competition of Oleic Acid with Dicarboxylic Acids for Binding

\begin{tabular}{|c|c|c|c|c|c|c|}
\hline \multirow[b]{2}{*}{ Fatty acid } & \multirow{2}{*}{$\begin{array}{c}\text { Bound } \\
\text { dicarboxylic } \\
\text { acid }\end{array}$} & \multicolumn{2}{|c|}{ Dissociation constants } & \multicolumn{2}{|c|}{ Predicted saturation } & \multirow[b]{2}{*}{ Number of data points } \\
\hline & & $K_{\mathrm{d}_{1}}$ & $K_{\mathrm{d}_{2}}$ & Site 1 & Site 2 & \\
\hline & $\mathrm{mol} / \mathrm{mol} \mathrm{BSA*}$ & \multicolumn{2}{|c|}{$\mu M \pm S D$} & \multicolumn{2}{|c|}{$\mathrm{mol} / \mathrm{mol} B S A \pm S D$} & \\
\hline $\begin{array}{l}\text { Hexadecanedioic } \\
\text { with }\end{array}$ & 4.4 & $1.2 \pm 1.4$ & $67.8 \pm 15.6$ & $0.9 \pm 0.3$ & $4.2 \pm 0.2$ & 51 \\
\hline $0.1 \mathrm{mM}$ oleic & 3.3 & & $41.0 \pm 7.3$ & & $3.7 \pm 0.1$ & 29 \\
\hline $0.3 \mathrm{mM}$ oleic & 1.4 & & $15.0 \pm 1.2$ & & $1.4 \pm 0.2$ & 14 \\
\hline $0.5 \mathrm{mM}$ oleic & 0.7 & & $34.4 \pm 9.6$ & & $0.8 \pm 0.1$ & 14 \\
\hline $\begin{array}{l}\text { Dodecanedioic } \\
\text { with }\end{array}$ & 0.9 & & $75.2 \pm 4.3$ & & $1.0 \pm 0.02$ & 28 \\
\hline $0.1 \mathrm{mM}$ oleic & 0.9 & & $339.3 \pm 116.0$ & & $1.4 \pm 0.2$ & 15 \\
\hline $0.5 \mathrm{mM}$ oleic & 0.4 & & $707.4 \pm 171.7$ & & $0.7 \pm 0.1$ & 22 \\
\hline
\end{tabular}

Competition of oleic acid with dicarboxylic acids for binding. * Indicates the mol ratio of fatty acid to albumin, as observed experimentally. 


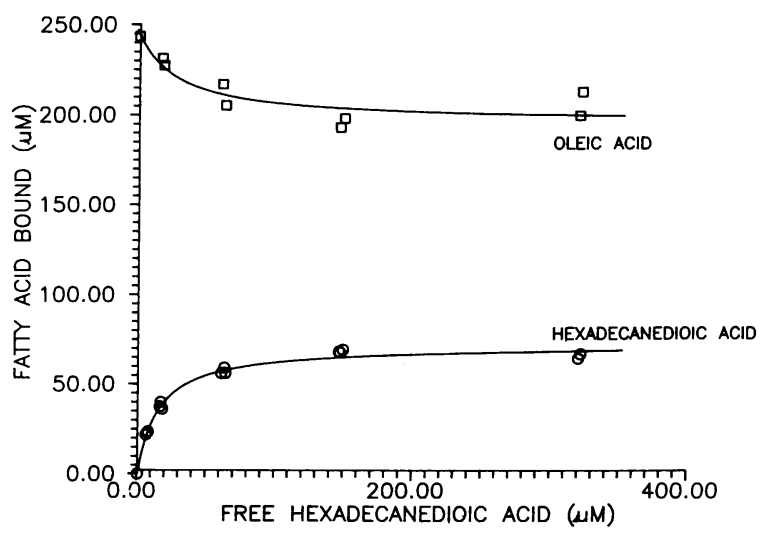

Figure 5. Competition of $0.3 \mathrm{mM}$ oleic acid with hexadecanedioic acid for binding to albumin. Binding was assessed as described in Fig. 4. The amount of bound oleate was determined after separation of free and bound fatty acid by centrifugation through a Sephadex G-25 column. $\square$, micromoles of bound oleic acid; $\bigcirc$, micromoles of bound hexadecanedioic acid. The binding of hexadecanedioic acid is analyzed using the equation of a Langmuir isotherm. The points are experimental data and the lines are the best fit of the data to the theoretical equations obtained using the nonlinear least-square computer program as described in the text. ${ }^{2}$

boxylic fatty acids of the same chain length. Spector and coworkers $(18,19)$ reported binding ratios of $6.5,7.2,7.0,8.4$, and 13.9 for $C_{18.0}, C_{16.0}, C_{14.0}, C_{12.0}$, and $C_{10.0}$, respectively compared with our observations of $3.8,4.2,1.6,0.8$, and 0.7 for dicarboxylic acids of the same chain length.

The $\mathrm{pH}$ of the buffer exerts only a modest effect on the binding of dicarboxylic acids. The $\mathrm{pK}$ of each of the carboxyl groups for octanedioic acid is 4.52 (26). The dissociation constants for longer chain dicarboxylic acids are probably close to this value. Thus, the dicarboxylic acids are $>99 \%$ deprotonated in the physiologic $\mathrm{pH}$ range we examined. The modest shift in binding at pH 6.8 may be due to a change in amino acid charge at one or more binding sites which facilitates binding of dicarboxylic acids.

As might be expected from a comparison of the dissociation constants of moncarboxylic and dicarboxylic acids, monocarboxylic acids competitively inhibit dicarboxylic acid binding. However, the competition studies demonstrate that at high enough concentrations, dicarboxylic acid can bind and displace some $\mathrm{C}_{18.1}$.

2. The binding of hexadecanedioic acid is analyzed using the equation of a Langmuir isotherm, $K_{d}^{\text {app }}=\left(S_{\mathrm{t}}^{\text {app }}-S_{\mathrm{b}}\right)\left(D_{\mathrm{f}}\right) / D_{\mathrm{b}}$, where $K_{\mathrm{d}}^{\text {app }}$ = apparent dissociation constant, $S_{t}^{\text {app }}=$ total sites on albumin available for binding hexadecanedioic acid under these experimental conditions, $S_{\mathrm{b}}=$ concentration of sites occupied by hexadecanedioic acid, $D_{\mathrm{b}}=$ concentration of bound hexadecanedioic acid, and $D_{\mathrm{f}}=$ concentration of free hexadecanedioic acid.

The desorption of oleic acid at various concentrations of hexadecanedioic acid is analyzed using the equation $K_{\mathrm{d}_{2}}=\left(S_{\mathrm{fd}}\right)\left(D_{\mathrm{f}}\right) / O_{\mathrm{bd}}=\left(O_{\mathrm{b}}\right.$ $\left.-D_{\mathrm{b}}{ }^{\max }\right)\left(D_{\mathrm{f}}\right) / O_{\mathrm{b}, \mathrm{d}=0}-O_{\mathrm{b}}$. In this equation, the total sites from which oleic acid can be displaced $=\mathrm{O}_{\mathrm{b}, \mathrm{d}=0}-D_{\mathrm{b}}{ }^{\max }$, where $O_{\mathrm{b}, \mathrm{d}=0}=$ oleic acid bound in the absence of hexadecanedioic acid, and $D_{b}{ }^{\max }=$ maximal hexadecanedioic acid able to bind to displaceable oleic acid sites on albumin. $D_{\mathrm{f}}=$ concentration of free hexadecanedioic acid, $O_{\mathrm{b}}=$ concentration of bound oleic acid, and $K_{\mathrm{d}_{2}}=$ dissociation constant $(\mathrm{M})$ for hexadecanedioic acid under these experimental conditions.

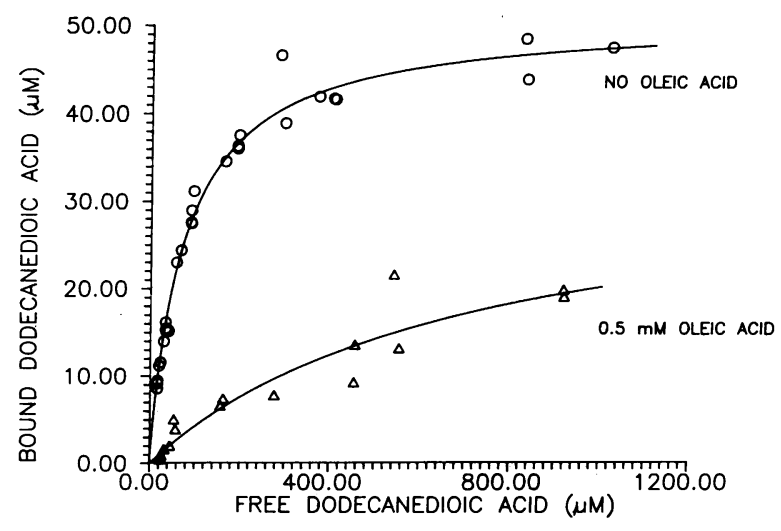

Figure 6. Competition of $0.5 \mathrm{mM}$ oleic acid with dodecanedioic acid for binding to albumin. Binding was assessed as described in Fig. 1. Binding in the presence of $0.5 \mathrm{mM}$ oleic acid $(\Delta)$ is compared with binding in the absence of competition ( 0 ).

The albumin molecule is composed of three nonidentical cylindrical domains (13), each of which has a narrow hydrophobic channel able to accomodate only one or two hydrocarbon chains. The ends of each domain form nonidentical subdomains containing positively charged amino acid side chains. Recent studies indicate that medium-chain monocarboxylic fatty acids bind almost exclusively via hydrophobic interactions, whereas the binding of long-chain monocarboxylic acids is dependent on both electrostatic and hydrophobic interac-

Table III. Competition of Dodecanedioic and Monocarboxylic Fatty Acids

\begin{tabular}{|c|c|c|c|}
\hline Concentration of fatty acids & $\begin{array}{c}\text { Bound } \\
\text { dodecanedioic }\end{array}$ & Bound oleic & $\begin{array}{c}\text { Bound } \\
\text { octanoic }\end{array}$ \\
\hline $\begin{array}{l}\mu M \text { dodecanedioic/ } \\
\mu M \text { oleic or octanoic }\end{array}$ & \multicolumn{2}{|c|}{$\mathrm{mol} / \mathrm{mol} \mathrm{BSA}$} & \\
\hline $10 / 0$ & 0.03 & - & - \\
\hline $200 / 0$ & 0.50 & - & - \\
\hline $300 / 0$ & 0.60 & 一 & - \\
\hline $0 / 150$ & - & 2.60 & - \\
\hline $10 / 150$ & 0.02 & 2.50 & - \\
\hline $200 / 150$ & 0.48 & 2.70 & - \\
\hline $300 / 150$ & 0.65 & 2.70 & - \\
\hline $0 / 320$ & - & 4.88 & - \\
\hline $10 / 320$ & 0.01 & 4.66 & - \\
\hline $200 / 320$ & 0.19 & 4.67 & - \\
\hline $300 / 320$ & 0.27 & 4.53 & - \\
\hline $0 / 100$ & - & - & 0.46 \\
\hline $200 / 100$ & 0.16 & - & 0.49 \\
\hline $300 / 100$ & 0.25 & - & 0.47 \\
\hline $0 / 500$ & - & - & 1.25 \\
\hline $200 / 500$ & 0.16 & - & 1.10 \\
\hline $300 / 500$ & 0.19 & - & 1.10 \\
\hline
\end{tabular}

Competition of dodecanedioic with the monocarboxylic acids octanoic, and oleic. Binding was assessed as described in Fig. 5. The concentration of albumin was $0.050 \mathrm{mM}$. 
tions (27). Three of the albumin subdomains are primary binding sites for long-chain monocarboxylic fatty acids. The other three subdomains are primary binding sites for medium- and short-chain fatty acids and drugs (13). Drugs bind along the rim of the subdomains through electrostatic interactions with positively charged amino acid groups.

The binding of dicarboxylic fatty acids to albumin resembles that of the monocarboxylic acids. Like medium-chain monocarboxylic fatty acids (13), the medium-chain dicarboxylic acids appear to bind to a single class of binding sites; the dissociation constants for these dicarboxylic acids are in the range of $10^{-5} \mathrm{M}$. The binding of the long-chain dicarboxylic acids resemble that of the long-chain monocarboxylic acids in that there are at least two classes of binding sites, one of relatively high affinity and one of low affinity. In the case of hexadecanedioic acid, for example, the $K_{d}$ for the primary binding site is $1.2 \mu \mathrm{M}$, and the $K_{\mathrm{d}}$ for the secondary binding sites is $68 \mu \mathrm{M}$.

There are, however, several important differences between the binding of monocarboxylic and dicarboxylic acids to albumin. First, the affinity of monocarboxylic acids for albumin is generally at least two orders of magnitude greater than that of dicarboxylic acids of the same chain length. Second, whereas there are three primary or high-affinity sites on albumin for long-chain monocarboxylic acids, there is only one such site for long-chain dicarboxylic acids. Third, whereas albumin binds up to $13 \mathrm{~mol}$ of medium-chain monocarboxylic acid per mol of albumin (19), only $1 \mathrm{~mol}$ of medium-chain dicarboxylic acid is bound per mol of albumin.

Our observations suggest that the single high-affinity site for long-chain dicarboxylic acids may be one of the high-affinity sites for monocarboxylic fatty acids. As shown in Table II, when the high-affinity monocarboxylic acid binding sites are largely occupied $\left(0.1 \mathrm{mM} \mathrm{C}_{18.1}\right)$, there appears to be only a single class of low-affinity sites for dicarboxylic acids, having a dissociation constant similar to that of the low-affinity sites in the absence of any competition. On the other hand, the lowaffinity sites for long- and medium-chain length dicarboxylic acids are distinct from the primary binding sites for long-chain monocarboxylic acids, as when these latter sites are occupied by monocarboxylic acids, all the low-affinity sites for hexadecanedioic and dodecanedioic acids are still available (Tables II and III); the dicarboxylic acids are only displaced when the bound $C_{18.1}$ exceeds $3 \mathrm{~mol} / \mathrm{mol}$ of albumin. Moreover, $C_{8.0}$ is more effective than $C_{18.1}$ (Table III) in inhibiting the binding at the low-affinity site. We infer from these considerations that some or all of the low-affinity sites for medium- and longchain dicarboxylic acids are in the subdomains of albumin that bind medium-chain monocarboxylic fatty acids and drugs.

These studies confirm our hypothesis that dicarboxylic acids are not as tightly bound to albumin as monocarboxylic fatty acids (9), and suggest that in Reye's syndrome, in which the fatty acid/albumin ratio approaches or exceeds $4: 1(4,28)$, substantial concentrations of dicarboxylic acids may be free. Long-chain dicarboxylic acids are readily displaced from the higher affinity binding site. Binding to the lower affinity sites is probably also significantly impaired in patients with Reye's syndrome. The concentration of dicarboxylic acids in those patients is often as high as $0.5 \mathrm{mM}$ in comatose patients, with the majority of the dicarboxylic acids being long chain (C14C18) (4). In our experiments, when the binding of $0.5 \mathrm{mM}$ hexadecanedioic acid was assessed in the presence of near satu- rating concentrations of $\mathrm{C}_{18.1}$, only $16 \%$ of hexadecanedioic acid was bound (Fig. 4). Moreover, Goodman and others (29, 25) have shown that when the fatty acid/albumin ratio exceeds $2: 1$, drugs, with affinity for albumin comparable to that which we observed for dicarboxylic acids, are largely unbound. Our studies suggest that the low-affinity sites for dicarboxylic acids are the same as the binding sites for medium-chain fatty acids and drugs, and thus may compete with these compounds for binding. Some investigators have, in fact, shown elevations in medium-chain fatty acids in Reye's syndrome (30). In addition, aspirin and other protein-bound medications are frequently found in patients with Reye's syndrome and have been suggested to play a role in the pathogenesis of the illness (9). Thus, it seems likely that with the competition of long- and medium-chain length monocarboxylic acids and drugs for binding to albumin, dicarboxylic acids are largely unbound in the plasma of patients with Reye's syndrome.

\section{Acknowledgments}

The authors thank E. van Mele, Francis Ko, and John O'Connell for their excellent technical assistance and Godfrey S. Getz, M.D., Ph.D. for advice and critical review of the manuscript.

The work was supported in part by the Children's Research Fund and the Jennifer Wieser Fund, the University of Chicago, a grant from the Markey Trust, and grants HD-04583, NS-23616, and HL-15062 from the United States Public Health Service.

\section{References}

1. Bjorkhem, I. 1978. On the quantitative importance of $\omega$-oxidation of fatty acids. J. Lipid Res. 19:585-590.

2. Tonsgard, J. H. 1985. Urinary dicarboxylic acids in Reye syndrome. J. Pediatr. 107:79-84.

3. Ng, K. J., B. D. Andresen, M. D. Hilty, and J. R. Bianchine. 1983. Identification of long chain dicarboxylic acids in the serum of two patients with Reye's syndrome. J. Chromatogr. 276:1-10.

4. Tonsgard, J. H. 1986. Serum dicarboxylic acids in Reye syndrome. J. Pediatr. 109:440-445.

5. Rocchiccioli, F., P. Aubourg, and P. F. Bougneres. 1986. Medium- and long-chain dicarboxylic aciduria in patients with Zellweger syndrome and neonatal adrenoleukodystrophy. Pediatr. Res. 20:6266.

6. Tanaka, K. 1979. Jamaican vomiting sickness. In Handbook of Clinical Neurology. Vol. 37. P. J. Vinken and G. W. Bruyn, editors. North-Holland Publishing Co., Amsterdam. 511-539.

7. Vianey-Liaud, C., P. Divry, N. Gregersen, and M. Mathieu. 1987. The inborn errors of mitochondrial fatty acid oxidation. $J$. Inherited Metab. Dis. 10:159-200.

8. DeVivo, D. C. 1978. Reye syndrome: a metabolic response to an acute mitochondrial insult. Neurology. 28:165-168.

9. Starko, K. M., G. Ray, L. B. Dominquez, W. L. Stromberg, and D. F. Woodall. 1980. Reye's syndrome and salicylate use. Pediatrics. 66:859-864.

10. Tonsgard, J. H., and G. S. Getz. 1985. Effect of Reye's syndrome serum on isolated chinchilla liver mitochondria. J. Clin. Invest. 76:816-825.

11. Passi, S., M. Picardo, M. Nazzaro-Porro, A. Breathnach, A. M. Cafaloni, and G. Serlupi-Crescenzi. 1984. Antimitochondrial effect of saturated medium chain length (C8-C13) dicarboxylic acids. Biochem. Pharmacol. 33:103-108.

12. Kimura, A. 1986. Morphological effect of organic acids on mitochondria. Acta. Paediatr. Jpn. 28:707-715.

13. Brown, J. R., and P. Shockley. 1982. Serum albumin: structure 
and characterization of its ligand binding sites. In Lipid-Protein Interactions. Vol. I. P. C. Jost and O. H. Griffith, editors. John Wiley \& Sons, New York. 25-68.

14. Lowe, J. B., J. C. Sacchettini, M. Laposta, J. J. McQuillan, and J. I. Gordon. 1987. Expression of rat intestinal fatty acid-binding protein in Escherichi coli. J. Biol. Chem. 262:5931-5937.

15. Spector, A. A. 1975. Fatty acid binding to plasma albumin. $J$. Lipid Res. 16:165-179.

16. Wojtczak, L. 1976. Effects of long chain fatty acids and acyl CoA on mitochondrial permeability, transport, and energy-coupling. J. Bioenerg. Biomed. 8:293-311.

17. Kritchevsky, D., L. M. Davidson, H. K. Kim, and S. Malhotra. 1973. Quantitation of serum lipids by a simple TLC-charring method. Clin. Chim. Acta. 46:63-68.

18. Spector, A. A., K. John, and J. E. Fletcher. 1969. Binding of long-chain fatty acids to bovine serum albumin. J. Lipid Res. 10:5667.

19. Ashbrook, J. D., A. A. Spector, and J. E. Fletcher. 1972. Medium chain fatty acid binding to human plasma albumin. J. Biol. Chem. 247:7038-7042.

20. Danielsson, I. 1956. The association of long-chain dipotassium $\alpha, \omega$-alkanedioates in aqueous solution. Acta Acad. Abo. Ser. B Math. Phys. Mat. Naturvefensk. Tek. 20:7-112.

21. Ockner, R. K., J. A. Manning, R. B. Poppenhausen, and W. K. L. Ho. 1972. A binding protein for fatty acids in cytosol of intestinal mucosa, liver, myocardium and other tissues. Science (Wash. DC). 177:56-58
22. Glatz, J. F., and J. H. Veerkamp. 1983. A radiochemical procedure for the assay of fatty acid binding by proteins. Anal. Biochem. 132:89-95.

23. Yamaoka, K., Y. Tanigawara, T. Nakagawa, and T. Una. 1981 A pharmacokinetic analysis program (multi) for microcomputers. $J$. Pharmacobiodyn. 4:879-885.

24. Adamson, A. W. 1982. Physical Chemistry of Surfaces. 4th ed. John Wiley \& Sons, New York. 521-523.

25. Spector, A. A., and J. E. Fletcher. 1978. Nutritional effects on drug-protein binding. In Nutrition and Drug Interrelations. J. N. Hathcock and J. Coon, editors. Academic Press, Inc., New York. 447-473.

26. Weast, R. C., and M. J. Astle, editors. 1981. CRC Handbook of Chemistry and Physics. CRC Press, Inc., Boca Raton, FL. D-143.

27. Cistola, D. P., D. M. Small, and J. A. Hamilton. 1987. Carbon 13 NMR studies of saturated fatty acids bound to bovine serum albumin. J. Biol. Chem. 262:10980-10985.

28. Pollack, J. D., M. D. Cramblett, D. Flynn, and D. Clark. 1975. Serum and tissue lipids in Reye's syndrome. In Reye's Syndrome. J. D. Pollack, editor. New York, Grune and Stratton, Inc., New York. 227-243.

29. Goodman, D. S. 1958. The interaction of human serum albumin with long-chain fatty acid anions. J. Am. Chem. Soc. 80:38923898.

30. Mamunes, P., G. H. DeVries, C. D. Miller, and R. B. David. 1974. In Reye's Syndrome. J. D. Pollack, editor. Grune and Stratton, Inc., New York. 245-254. 